\title{
Cierre secuencial de la pared abdominal en el manejo del abdomen abierto. Una nueva técnica quirúrgica
}

\author{
Sequential closure of the abdominal wall for the management of open abdomen. A new \\ surgical technique
}

Efrén Flores-Álvarez*, J. Cruz De la Torre-González, Virgilio Rivera-Barragán y Luis D. De la Cruz-Álvarez Departamento de Enseñanza e Investigación y Servicio de Cirugía General, Centenario Hospital Miguel Hidalgo, Aguascalientes, México

\begin{abstract}
Resumen
Antecedentes: El abdomen abierto es un método quirúrgico utilizado en el tratamiento de pacientes con sepsis abdominal, en trauma abdominal en casos de cirugía de control de daños y en casos de síndrome de hipertensión abdominal. Objetivo: Demostrar la efectividad de una nueva técnica quirúrgica en pacientes con abdomen abierto. Método: Estudio de todos los pacientes manejados con abdomen abierto en nuestro hospital en un periodo de 5 años. Resultados: Se incluyeron 24 pacientes, 18 hombres y 6 mujeres, con una edad promedio de $41.5 \pm 15.9$ años. El diagnóstico operatorio fue síndrome compartimental abdominal en 7 (29\%) casos, sepsis abdominal en 9 (38\%) y trauma abdominal en 8 (33\%). La puntuación APACHE II tuvo una mediana de 8 (rango: 5-21) y el SIRS una mediana de 2 (rango: 1-4). La mediana de ingresos a quirófano por paciente fue de dos. La mediana de las aproximaciones aponeuróticas fuera de quirófano fue de cuatro. Se realizó un cierre definitivo de la pared abdominal en 21 de 22 pacientes vivos, considerando cierre exitoso en el 95\%. Conclusiones: El cierre secuencial es una técnica efectiva que ofrece una alternativa en pacientes que requieren manejo con abdomen abierto.
\end{abstract}

Palabras clave: Abdomen abierto. Hipertensión abdominal. Pared abdominal. Sepsis abdominal. Trauma abdominal.

\begin{abstract}
Background: The open abdomen is a surgical technique used in the treatment of patients with abdominal sepsis, abdominal trauma and abdominal hypertension syndrome. Objective: The aim was to demonstrate the effectiveness of a new surgical technique designed for the management and closure of the abdominal wall in patients with open abdomen. Method: Study of all patients treated with open abdomen in our Hospital over a five-year period. Results: It were included 24 patients, 18 men and 6 women. The average age was $41.5 \pm 15.9$ years. Operative diagnosis was abdominal compartment syndrome in 7 (29\%) cases, abdominal sepsis in 9 (38\%), and abdominal trauma in 8 (33\%). The median of APACHE II score was 8 points (range: 5-21) while the assessment of SIRS score had a median of 2 points (range: 1-4). The median of surgical procedures performed in operating room was two per patient. The median of fascial surgical closures performed in the patient bed was four. A successful closure of the abdominal wall was performed in 21 of 22 live patients (95\%). Conclusions: The sequential closure of the abdominal wall is an effective technique that offers an alternative to the management of the open abdomen.
\end{abstract}

Key words: Open abdomen. Abdominal hypertension. Abdominal wall. Abdominal sepsis. Abdominal trauma.

\section{Correspondencia:}

*Efrén Flores Álvarez

Avda. Gómez Morín, s/n

Col. La Estación, La Alameda

Fecha de recepción: 24-09-2019

Cir Cir. 2020;88(5):624-629

C.P. 20259, Aguascalientes, Ags, México

Fecha de aceptación: 15-05-2020

Contents available at PubMed

E-mail: efflorez@ hotmail.com

DOI: 10.24875/CIRU.20000760

www.cirugiaycirujanos.com

0009-7411/@ 2020 Academia Mexicana de Cirugía. Publicado por Permanyer. Este es un artículo open access bajo la licencia CC BY-NC-ND (http://creativecommons.org/licenses/by-nc-nd/4.0/). 


\section{Introducción}

El abdomen abierto es una estrategia quirúrgica que ha resultado del avance en el manejo operatorio del paciente con infección intraabdominal complicada y desde recientemente se emplea en pacientes con traumatismo abdominal grave que requieren laparotomía abreviada o de salvamento, así como en la prevención y el tratamiento del síndrome de hipertensión abdominal' ${ }^{1}$.

Se han descrito múltiples cuadros en los cuales la infección, la irritación peritoneal, la presencia de sangre o de compresas en la cavidad abdominal, la reanimación agresiva con líquidos o el cierre de un gran defecto de la pared van a condicionar edema visceral y retroperitoneal, con un consecuente incremento en la presión intraabdominal, la cual puede alcanzar niveles tan peligrosos que ocasione en algunos casos una disfunción orgánica sistémica. Se ha demostrado que los pacientes en estas condiciones tienen una mejor evolución si son tratados con abdomen abierto temporalmente, permitiendo una mejor perfusión visceral abdominal y una menor repercusión del funcionamiento sistémico ${ }^{2,3}$.

El abdomen abierto, si bien ofrece diversos beneficios, también crea numerosos riesgos de alta morbilidad para los pacientes, tales como alteraciones hidroelectrolíticas, contaminación de órganos abdominales, desarrollo de fístulas enteroatmosféricas, adherencias obstructivas y defectos herniarios gigantes de la pared abdominal con consecuencias estéticas y funcionales importantes ${ }^{4-6}$.

En la actualidad hay un notable aumento del empleo de las técnicas para el cierre del abdomen abierto, y se han ideado nuevos procedimientos que además de resolver el evento agudo en forma satisfactoria intentan evitar las serias complicaciones inherentes al manejo a largo plazo del paciente con abdomen abierto. El cierre secuencial de la pared abdominal (CSPA) es una técnica ideada y desarrollada en el Centenario Hospital Miguel Hidalgo, en la que se emplean materiales y métodos utilizados en diversas técnicas para el manejo y el cierre del abdomen abierto, con la particularidad de utilizar instrumentos y material accesible y disponible en cualquier sala de quirófano de un hospital de segundo o tercer nivel de atención.

\section{Método}

Se realizó un estudio prospectivo, transversal, descriptivo y analítico que incluyó a todos los pacientes adultos tratados con abdomen abierto en el servicio de cirugía general entre julio de 2004 y julio de 2012. Las variables estudiadas fueron edad, sexo, estado nutricional, causa, días de estancia hospitalaria y en la unidad de terapia intensiva, número de aproximaciones de la aponeurosis, presión intraabdominal durante el manejo, puntaje APACHE II (Acute Physiology And Chronic Health Evaluation II) y síndrome de respuesta inflamatoria sistemica (SRIS), complicaciones y mortalidad.

\section{Técnica quirúrgica}

Para efectuar la técnica de CSPA se utiliza una interfase de polietileno que puede ser una bolsa recolectora de orina o una bolsa de plástico fijada con puntos cardinales de poliéster al espesor total de la pared abdominal, cuya finalidad es contener las asas intestinales y permitir el deslizamiento medial progresivo de la pared abdominal. Es necesaria una disección amplia del borde de la aponeurosis separándola del tejido celular subcutáneo, creando el suficiente espacio para colocar puntos aponeuróticos de aproximación por medio de tubos de drenaje cerrado de 1/8" (Drenovac ${ }^{\circledR}$ ) o catéteres intravenosos largos sin retirar la guía de acero. Enseguida se realiza el afrontamiento de los bordes aponeuróticos, manteniendo cierta tensión utilizando pinzas de Kelly o mediante un sistema de cubos de acrílico con tornillos de ajuste, diseñados en el Centenario Hospital Miguel Hidalgo por los autores del estudio. En algunos pacientes se colocan una compresa estéril y una sonda de aspiración para drenar el contenido peritoneal.

A través de la medición de la presión intraabdominal y de la evolución clínica del paciente se determina la frecuencia con la que se ajusta el afrontamiento; generalmente se realizan ajustes cada 24-48 horas. Se requieren entre dos y cuatro ajustes para lograr la aproximación completa de los bordes de la aponeurosis. Los ajustes se efectúan en la cama del paciente y el cierre definitivo de la pared se realiza en el quirófano.

Se decide cerrar en forma definitiva la pared abdominal cuando la presión abdominal es menor de $15 \mathrm{mmHg}$, las fascias se han aproximado lo suficiente para permitir un cierre seguro de la pared abdominal, existe la seguridad de que no hay fugas intestinales, la continuidad intestinal ha quedado restablecida, se ha logrado la debridación suficiente de tejido necrótico y la infección intraabdominal ha sido resuelta. 


\section{Análisis estadístico}

Se realizó un análisis descriptivo de cada una de las variables. Para las variables cualitativas se calcularon las frecuencias absolutas y los porcentajes. Para las variables cuantitativas se estimaron el promedio y la desviación estándar, o bien la mediana y los rangos, según el tipo de distribución de los datos.

\section{Resultados}

Se incluyeron 24 pacientes, de los cuales seis eran mujeres (25\%) y 18 eran hombres (75\%). La media de edad fue de $41.5 \pm 15.9$ años. Todos los pacientes fueron inicialmente operados de urgencia, y los diagnósticos preoperatorios fueron: cuadro abdominal agudo en $16(67 \%)$ y trauma abdominal en 8 (33\%); en seis fue un trauma abdominal contuso y en uno fue penetrante. Ocho pacientes (33\%) fueron manejados inicialmente con abdomen abierto. La causa directa que motivó el manejo mediante la técnica de la «bolsa de Bogotá» fue la imposibilidad de realizar un cierre de la aponeurosis sin tensión; los restantes $16(67 \%)$ pacientes fueron relaparatomizados e ingresados al estudio debido al desarrollo de síndrome compartimental o sepsis abdominal.

Todos los pacientes fueron valorados y protocolizados por los autores del estudio para la realización del CSPA. En la evaluación preoperatoria para la instalación del sistema, los pacientes tuvieron un puntaje de APACHE II con una mediana de 8 puntos (rango: 5-21 puntos) y un SRIS con una mediana de 2 (rango: 1-4) puntos. Ocho de los 24 pacientes (33\%) se consideraron desnutridos según la escala de valoración nutricional global subjetiva. Los valores de los parámetros nutricionales bioquímicos fueron los siguientes: albúmina, mediana de $2.15 \mathrm{mg} / \mathrm{dl}$ (rango: 1.03-3.8), y linfocitos totales, mediana de 1,820 (rango: 730-3750). La mediana de la hemoglobina de los pacientes a su ingreso fue de $13.9 \mathrm{~g} / \mathrm{dl}$ (rango: 9.2-15.8) y la mediana del hematocrito fue del 39.5\% (rango: 24-47.6).

Se efectuó la técnica quirúrgica de CSPA a los 24 pacientes. En 17 casos (71\%), el afrontamiento de las aponeurosis se realizó empleando tubos de drenaje y en 7 pacientes (29\%) catéteres intravenosos. El promedio de ingresos a quirófano por paciente fue de 2 (rango: 1-5). La mediana del número de aproximaciones aponeuróticas en la cama del paciente fue de 4 (rango: 3-7), siempre precedidas por la medición de la presión intraabdominal.
El monitoreo de la presión intraabdominal fue estricto en todos los pacientes: a las 8 horas del evento quirúrgico, la mediana fue de $14 \mathrm{cmH}_{2} \mathrm{O}$ (rango: 9-21); a las 12 horas fue de $14 \mathrm{cmH}_{2} \mathrm{O}$ (rango: 8-18); a las 24 horas fue de $10 \mathrm{cmH}_{2} \mathrm{O}$ (rango: 6-14); a las 48 horas fue de $9 \mathrm{cmH}_{2} \mathrm{O}$ (rango: 4-14 ); a las 72 horas fue de $8 \mathrm{cmH}_{2} \mathrm{O}$ (rango: 5-13); inmediatamente previo al cierre definitivo de la pared abdominal fue de $7 \mathrm{cmH}_{2} \mathrm{O}$ (rango: 4-11); y a las 24 horas del cierre definitivo fue de $12 \mathrm{cmH}_{2} \mathrm{O}$ (rango: 4-16).

Nueve de los 24 (38\%) pacientes fueron tratados en la unidad de terapia intensiva, con una media de estancia en dicho servicio de $13.5 \pm 6$ días. Diez pacientes $(42 \%)$ recibieron apoyo mecánico ventilatorio por un promedio de $11 \pm 3$ días. Los días de estancia hospitalaria fueron en promedio $29 \pm 14$ días.

Se consideró un cierre definitivo de la pared abdominal en 21 pacientes (88\%). El procedimiento completo fue fallido en tres casos (13\%), debido a que dos pacientes fallecieron antes de realizar el cierre definitivo de la pared abdominal y a otro se le retiró el sistema para colocar una «bolsa de Bogotá» debido al desgarro en varios puntos de la aponeurosis. En los supervivientes al evento agudo se logró un cierre exitoso en 21 de 22 pacientes (95\%). En total, fallecieron 4 pacientes (17\%).

Durante el seguimiento, 3 pacientes han desarrollado una hernia ventral incisional, de 2, 3 y $5 \mathrm{~cm}$; las últimos dos reparadas en forma electiva y ambulatoria, sin mayores complicaciones.

\section{Discusión}

El empleo de la técnica quirúrgica de abdomen abierto va en aumento al mismo tiempo que los cirujanos se familiarizan con el procedimiento, la selección de los pacientes, el equipo y los instrumentos empleados, el funcionamiento y las posibles disfunciones del sistema, los efectos benéficos y las desventajas que representa su uso en la práctica clínica. El abdomen abierto es en la actualidad el procedimiento quirúrgico estándar empleado para pacientes sometidos a cirugía de control de daños, en la descompresión en casos de síndrome compartimental abdominal y en el manejo de la sepsis abdominal grave. Este tratamiento ha salvado muchas vidas, pero ha creado nuevos problemas que debe enfrentar el cirujano, tales como la pérdida de líquidos y proteínas, el desarrollo de fístulas intestinales y la retracción del borde de las fascias musculares de la pared muscular, con la consecuente formación de hernias ventrales gigantes? 


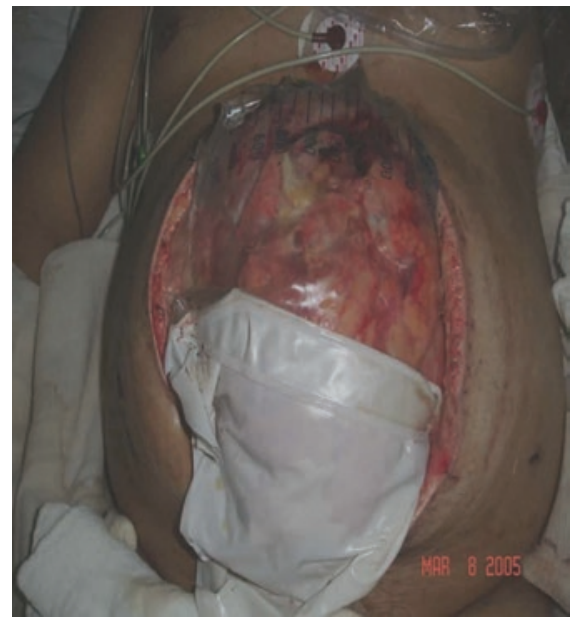

Figura 1. Paciente con abdomen abierto y «bolsa de Bogotá».

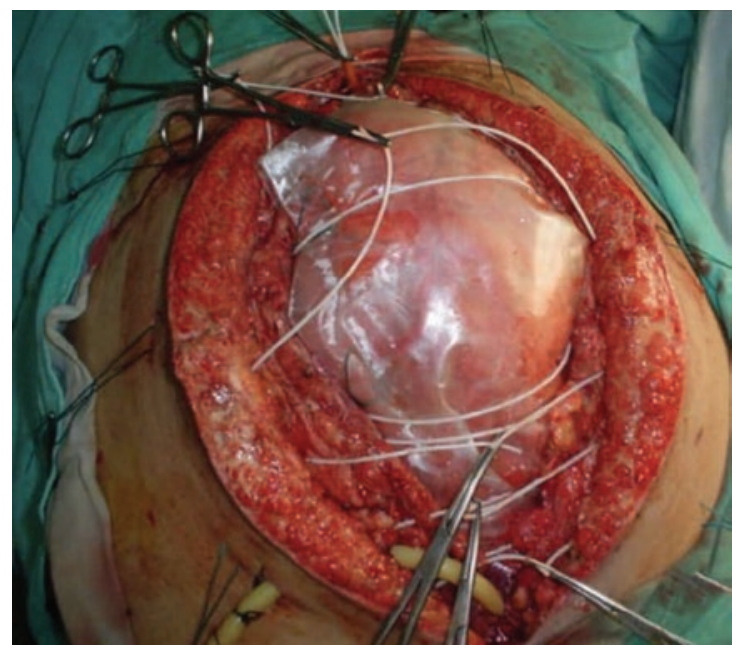

Figura 2. Cierre secuencial de la pared abdominal. Se muestran la interfase de polietileno y la fijación de los catéteres de retracción insertados en la aponeurosis.

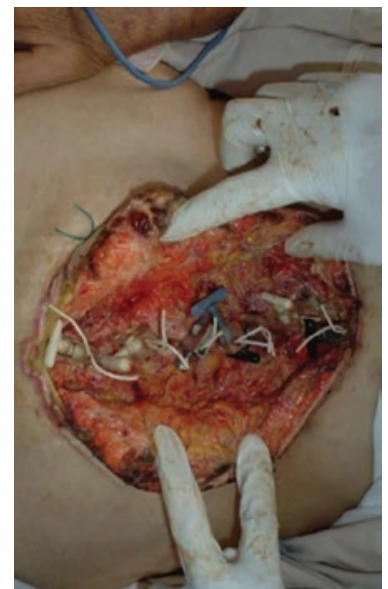

Figura 3. Afrontamiento progresivo de las aponeurosis manteniendo la tracción mediante cubos acrílicos diseñados en el hospital.

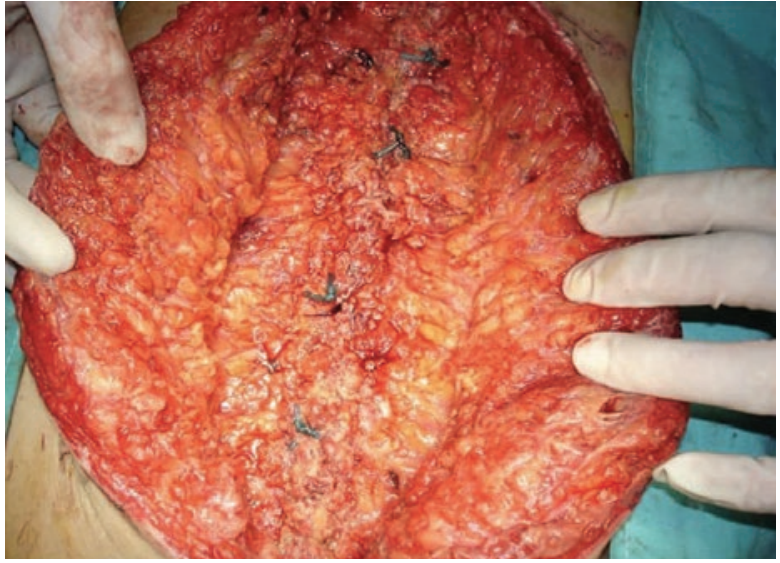

Figura 4. Cierre definitivo de la pared suturando los bordes de la aponeurosis.

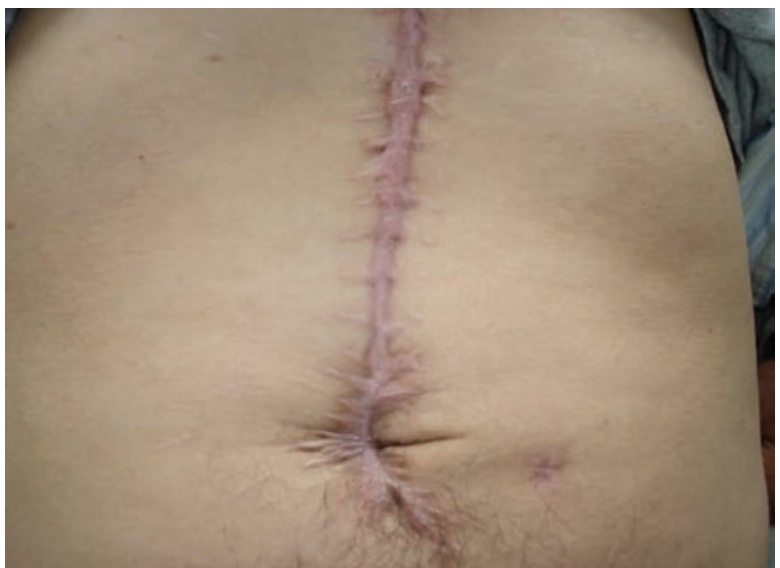

Figura 5. Resultado final en el abdomen de un paciente tratado con cierre secuencial de la pared abdominal.

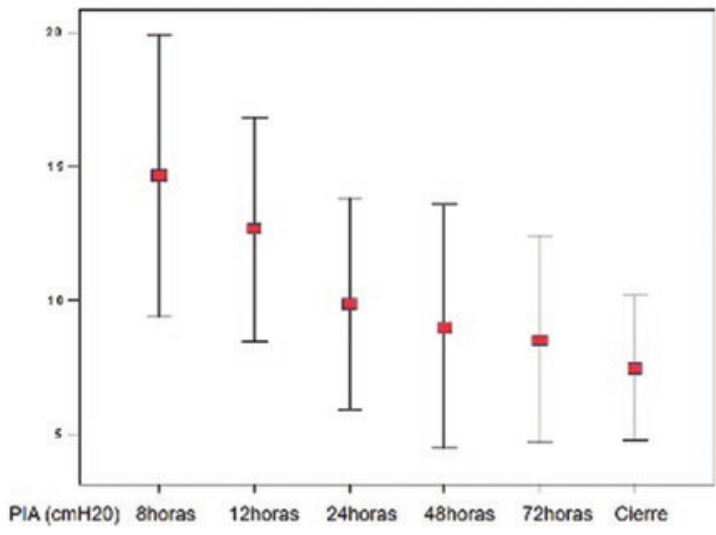

Figura 6. Promedio de la determinación de la presión intraabdominal (PIA) durante el procedimiento. 
En nuestro hospital son habituales los pacientes que requieren manejo con abdomen abierto para prevenir o tratar el síndrome compartimental, en la reexploración planificada por sepsis peritoneal 0 en la cirugía de control de daños. Durante muchos años, el grupo de cirujanos ha utilizado la técnica de la «bolsa de Bogotá» como un procedimiento estándar y ha intentado realizar de forma aislada, parcial y no protocolizada, algunos de los diferentes métodos reportados para el cierre de la pared abdominal, que en su mayoría son técnicas quirúrgicas que implican el uso de materiales e instrumental de alto costo, por lo que su empleo en nuestro medio es muy limitado y difícil de adoptar como método convencional.

La mayoría de los pacientes que requieren este tipo de manejo son adultos jóvenes, económicamente activos y con larga expectativa de vida, por lo que los defectos cosméticos y funcionales de la pared abdominal son de gran relevancia ${ }^{8}$.

En nuestra serie de pacientes todos eran jóvenes y la causa fue de origen traumático o infeccioso.

La principal indicación del abdomen abierto y del CSPA al momento de la cirugía se presenta en pacientes con un proceso abdominal agudo de tipo infeccioso cuando los bordes aponeuróticos no pueden ser afrontados sin tensión debido al edema y la distensión de las asas intestinales; sin embargo, puede emplearse en diversas circunstancias, cuando la lesión intraabdominal no puede ser resuelta adecuadamente en la intervención primaria, cuando el paciente esta inestable o muy grave para realizar una operación extensa y es necesaria una cirugía de control de daños, cuando la viabilidad del intestino es cuestionable y requiere una segunda intervención (conocida como cirugía de segunda mirada o second look), cuando se efectúa una anastomosis primaria en circunstancias críticas con una alta probabilidad de dehiscencia, ante una hemorragia de difícil control que requiere empaquetamiento o después de una operación prolongada con extensa exposición de vísceras que ocasiona edema peritoneal por exposición ${ }^{3,6,9}$. En la sepsis abdominal, la técnica de abdomen abierto proporciona además un acceso a la cavidad peritoneal para desbridación de tejido desvitalizado, aspiración de material purulento y drenaje quirúrgico ${ }^{10}$.

La evolución natural de los pacientes que son manejados con la «bolsa de Bogotá» es hacia el desarrollo de una hernia ventral planeada y grandes cicatrices ocasionadas por el cierre de la herida por segunda intención. En estos pacientes, en el mejor de los casos se intenta una reparación mucho tiempo después mediante procedimientos complejos que incluyen el uso de material protésico de gran tamaño, con elevada morbilidad y un elevado índice de fracasos ${ }^{11}$. Al igual que con la mayoría de las técnicas de cierre progresivo, con el CSPA se conserva la integridad de los componentes de la pared abdominal y es posible realizar el cierre definitivo sin el uso de materiales protésicos permanentes.

Actualmente sabemos que el edema visceral y retroperitoneal que ocurre en el estado de choque y la reperfusión tisular pueden incrementar la presión de la cavidad abdominal a niveles peligrosos, ocasionando una disfunción orgánica sistémica. Se ha demostrado que los pacientes en estas condiciones tienen una mejor evolución si son tratados con abdomen abierto, permitiendo una mejor perfusión visceral abdominal ${ }^{12}$. Además, se ha demostrado que un cierre forzado en condiciones de tensión de la pared abdominal puede ocasionar isquemia, necrosis, tendencia a la infección y dehiscencia de la pared abdominal ${ }^{13}$. Los beneficios del CSPA son reparar, drenar, descomprimir y ofrecer un control de la pared abdominal en aquellos pacientes que requieren tratamiento con abdomen abierto. La técnica tiene la ventaja de que el paciente puede permanecer en su cama, sin necesidad de los reiterados ingresos a quirófano requeridos por otros métodos. Debido a que se trata de una técnica sencilla y fácil de realizar, así como por el uso de materiales inertes, se asocia a un bajo índice de complicaciones ${ }^{14}$.

Consideramos que el cierre secuencial de la pared abdominal es una técnica adecuada para el manejo de los pacientes que requieren tratamiento mediante abdomen abierto. Con los resultados obtenidos podemos afirmar que es un procedimiento que cumple fielmente con los objetivos más ambiciosos del cierre progresivo de la pared abdominal, y que es una técnica segura y eficaz que puede ser reproducida en cualquier hospital de segundo o tercer nivel de atención.

\section{Conclusión}

EI CSPA es una técnica quirúrgica sencilla y fácil de realizar, ideada y empleada en el Centenario Hospital Miguel Hidalgo de manera protocolizada y estandarizada, que ofrece una alternativa para el manejo de los pacientes con abdomen abierto, con resultados iniciales alentadores.

\section{Agradecimientos}

A todos los integrantes del departamento de cirugía del Centenario Hospital Miguel Hidalgo, médicos adscritos al servicio de cirugía general, médicos 
residentes e internos de pregrado, enfermeras y Bibliografía

trabajadoras sociales.

\section{Conflicto de intereses}

Los autores declaran que no existe ningún conflicto de intereses.

\section{Financiamiento}

Este estudio no ha recibido financiamiento externo.

\section{Responsabilidades éticas}

Protección de personas y animales. Los autores declaran que para esta investigación no se han realizado experimentos en seres humanos ni en animales.

Confidencialidad de los datos. Los autores declaran que han seguido los protocolos de su centro de trabajo sobre la publicación de datos de pacientes.

Derecho a la privacidad y consentimiento informado. Los autores han obtenido el consentimiento informado de los pacientes y/o sujetos referidos en el artículo. Este documento obra en poder del autor de correspondencia.

1. Lossanoff JE, Richman BW, Jones JW. Temporary abdominal coverage and reclosure of the open abdomen: frecuently asked questions. J Am Coll Surg. 2002;195:105-15.

2. Eddy VA, Key SP, Mirris JA. Abdominal compartment syndrome: etiology, detection, and management. J Tenn Med Assoc. 1994;87:55-7.

3. Flores-Álvarez E, Ávila-Cuevas GE, López-Rodríguez JL, Reynoso-Talamantes D, De la Torre-González J, Rivera-Barragán V. Detección temprana y factores de riesgo asociados al síndrome compartimental abdominal. Cir Cir. 2005;73:179-83.

4. Arenas-Márquez $\mathrm{H}$. Manejo del abdomen abierto. Los mejores resultados. Cir Gen. 2010;32:S43-45.

5. Borráez O. Abdomen abierto. Utilización del polivinilo. Rev Colomb Cir. 2001;16:39-43.

6. Kreis BE, de Mol van Otterloo AJ, Kreis RW. Open abdomen management: A review of its history and proposed management algorithm. Med Sci Monit. 2013;19:524-33.

7. Demetriades D, Salim A. Management of the open abdomen. Surg Clin North Am. 2014;94:131-53.

8. Ivatury RR. Update on open abdomen management: achievements and challenges. World J Surg. 2009;33:1150-3.

9. Tavares-de la Paz LA, Andrade-de la Garza P, Goné-Fernández A, Sánchez-Fernández P. Abdomen abierto. Evolución en su manejo. Cir Cir. 2008;76:177-86.

10. Schecter WP, Ivatury RR, Rotondo MF, Hirshberg A. Open abdomen after trauma and abdominal sepsis: a strategy for management. J Am Coll Surg. 2006;203:390-6.

11. Villa-Sánchez MA, Álvarez F, Roa-Rossi A. Reparación de hernia ventral consecuente a laparostomía. Rev Colomb Cir. 2004;19:156-61.

12. García-Ureña MA, López-Monclús J, Robín A. Surgical analysis of the new clinical practice guide on compartmental syndrome. Med Intensiva. 2014:38:170-2.

13. Yuan $Y$, Ren J, He Y. Current status of the open abdomen treatment for intraabdominal infection. Gastroenterol Res Pract. 2013;2013:1-7.

14. Flores-Álvarez E, Rivera-Barragán V, De la Torre-González J. Cierre secuencial de la pared abdominal. Una nueva técnica quirúrgica diseñada en el Centenario Hospital Miguel Hidalgo para el manejo del abdomen abierto. Lux Médica. 2010;5:15-22. 